\title{
Liver biopsy in the diagnosis of cirrhosis
}

Many diagnostic procedures are now available for the assessment of the cirrhotic patient; for example, measurement of serum antibodies against smooth muscle and mitochondria helps in the recognition of active chronic hepatitis and primary biliary cirrhosis, and an important complication of cirrhosis, primary liver cell carcinoma, is more readily diagnosed with the aid of coeliac axis arteriography, liver scintiscanning, and estimation of $\alpha$-fetoglobin in the patient's serum. In spite of the use of such modern methods, liver biopsy continues to play a part in the diagnosis and management of cirrhotic patients, and concepts of the disease remain firmly based on morphology. This article is an attempt to summarize the different uses of percutaneous biopsies in cirrhotic patients and to review the problems of interpretation caused by the provision of a sample representing perhaps one hundred thousandth of the whole organ.

There is much disagreement on the value of percutaneous, blind needle biopsies in establishing a diagnosis of cirrhosis. Braunstein ${ }^{1}$ took multiple needle samples from cirrhotic livers at necropsy, using a Vim-Silverman needle. Of 507 samples only 10 failed to reveal cirrhosis. The accuracy of diagnosis in posthepatitic and postnecrotic cirrhosis was $96 \%$, that in 'nutritional' cirrhosis $100 \%$. The author concluded that if a biopsy failed to show fibrosis or cirrhosis, a diagnosis of cirrhosis could be ruled out with reasonable certainty. This view is supported by Baggenstoss ${ }^{2}$ who found liver biopsy a good method of establishing a morphological diagnosis of cirrhosis, though less helpful in determining its cause. Wilber and Faulk stated that a morphological diagnosis of cirrhosis was rarely in error, but that this was of relatively little help to the clinician. On the other hand Vido and Wildhirt, ${ }^{4}$ expressing a view prevalent on the continent of Europe, prefer laparoscopy as a method of diagnosing cirrhosis. In their series of 272 patients with cirrhosis blind biopsy failed to establish the diagnosis in just over half, and these authors regard liver biopsy as more useful in following the progress of the lesion than in establishing its presence. These differences are partly explained by the fact that the widely used Menghini needle tends to glance off the hard fibrous tissue and selectively samples the softer parenchyma, a problem largely avoided by the cutting action of the VimSilverman needle. There has been an attempt to overcome this problem by the use of newer needles having some of the advantages of both. ${ }^{5}$ Whatever the type of needle used, accurate diagnosis of cirrhosis in biopsies depends on an understanding of the morphological changes, and on the differentiation of cirrhosis from fibrosis; in any liver with extensive fibrosis a few peninsulas of parenchyma will be seen as islands in cross section, and may lead to a wrong diagnosis of hyperplastic nodules and hence cirrhosis.

In the majority of cases of cirrhosis, in most parts of the world, aetiology cannot be determined from the histological appearances. In others, features of alcoholic liver disease, biliary obstruction, venous outflow obstruction, or haemochromatosis can be recognized with reasonable certainty. Areas of intense activity showing liver cell destruction, rosette formation, and plasma cell infiltration suggest that the cirrhosis may be the end result of active chronic hepatitis, but this is itself of unknown aetiology. In active chronic 
hepatitis such active areas often alternate with large and relatively featureless hyperplastic nodules, so that the characteristic features can be missed on account of sampling error, the most important problem facing the interpreter. Primary biliary cirrhosis is probably more common than was first supposed, and can present other than with chronic cholestatic jaundice and itching. ${ }^{6,7}$ The classical lesions involve the larger interlobular and septal bile ducts, and as these are not always included in a needle biopsy the diagnosis can easily be missed; multiple sections of a biopsy may have to be examined before bile duct lesions or granulomas are found. Later in the course of the disease lesions are more widespread but at the same time less characteristic. Piecemeal necrosis of liver cells, the gradual destruction of liver cells at the expense of the adjacent mesenchyme, is common in primary biliary cirrhosis so that confusion with active chronic hepatitis and other forms of chronic hepatocellular disease may occur. In all forms of cirrhosis, except perhaps untreated haemochromatosis, recognition of the cause becomes more difficult with the passage of time and there is a tendency for an end-stage liver to develop, analogous to the granular contracted kidney which follows a number of different renal diseases. An example of this evolution of cirrhosis is seen in alcoholic liver disease. Classically the cirrhotic liver in chronic alcoholics is large, with small nodules rich in fat, separated by narrow septa and showing features of acute alcoholic hepatitis. With the passage of time, however, repeated episodes of necrosis lead to broadening of septa, areas of collapse and increased liver cell hyperplasia, and the cirrhosis thus changes into one of 'postnecrotic' type. ${ }^{8}$ It has been shown that in such cases there is a greater risk of primary liver cell carcinoma. ${ }^{9}$

Needle biopsy may help to establish the anatomical type of cirrhosis although sampling error tends to make this difficult. However, knowledge of the different types or stages of cirrhosis is essential if errors in interpretation are to be avoided. Since aetiology is often uncertain and pathogenesis difficult to establish, it is now customary in many centres to subdivide cirrhosis according to readily observable anatomical features. Thus Popper and Schaffner ${ }^{10}$ refer to 'regular' and 'irregular' cirrhosis, while others use the terms 'micronodular', 'macronodular', and 'mixed' cirrhosis.11,12 These have tended to replace the older and unsatisfactory classification into 'postnecrotic', 'portal', and 'biliary' cirrhosis. The term 'postnecrotic' is redundant in that all cirrhosis follows liver cell injury and misleading in that a preceding episode of massive necrosis is rare; 'portal' cirrhosis has little to do with portal tracts or veins and biliary obstruction more often causes fibrosis than true cirrhosis. The application of a simple anatomical description should not, however, lull the observer into believing that he has adequately characterized the disease, and it has already been observed that anatomical features change in the course of the disease and that therefore anatomical types represent stages in its evolution rather than separate entities. Meister, Szanto, and Schoolman ${ }^{13}$ failed to separate cirrhotic livers into sharply different populations on the basis of nodule size and appearance and concluded that there was no natural borderline between 'postnecrotic' and 'portal' cirrhosis. There is an infinite variation in the appearances of cirrhotic livers with respect to size, type of nodule, and scar tissue, depending on the interplay of liver cell death, liver cell hyperplasia, mesenchymal proliferation, and the storage of fat or iron.

A close relationship exists between the anatomical type and the ease with which cirrhosis can be diagnosed in a needle biopsy. In micronodular or regular cirrhosis the nodules are of the same order of size as the original lobules, and the lesions are regularly distributed throughout the liver. The lesion is therefore likely to be apparent in any needle biopsy of reasonable size. In macronodular or irregular cirrhosis, on the other hand, nodule diameter is often greater than the length of a needle biopsy and the archi- 
tecture of the nodules may closely resemble that of normal liver. This is especially so in the case of the incomplete septal or 'posthepatitic' variety ${ }^{14,15}$ in which slender septa demarcate large nodules. Because of this difficulty, it is often impossible to exclude cirrhosis entirely by needle biopsy, and diagnosis requires close attention to minor alterations in structure, most easily seen in sections impregnated with silver to demonstrate reticulin fibres. Features which support a diagnosis of macronodular cirrhosis include fragmentation of the biopsy before or during processing in the laboratory, fibrosis, especially when seen as a thin band of fibres at the edge of rounded pieces of liver tissue, structural abnormalities such as unusual relationships between afferent and efferent vessels and excess of the latter, marked variation in liver cell appearance, evidence of regeneration, and absence of lipofuscin pigment.

The problem of assessing the stage which cirrhosis has reached has already been raised in considering different anatomical types. In the case of micronodular cirrhosis, in which sampling error plays a smaller part than in other forms, an impression may be gained that cirrhosis is at an early stage of development on the one hand, or fully developed on the other. In the former case nodules are not well demarcated and it is difficult to decide whether there is fibrosis or cirrhosis. This situation, not uncommon in chronic alcoholics, is of some importance, as the lesion may be arrestable or even to some extent reversible in the early stages.

The activity of cirrhosis is a measure of its rate of progression, as judged histologically by the extent of liver cell necrosis and consequent inflammatory response. Necrosis may be of the type associated with the initial injury, for example, alcoholic or viral hepatitis; alternatively it may take the form of piecemeal necrosis. This does not always correlate well with biochemical and clinical evidence of activity, probably because cirrhosis may vary in activity in different parts of the liver and a small sample is not necessarily representative. It follows that minor variations in activity in consecutive biopsies in one individual patient cannot be taken as proof of the efficacy or otherwise of a mode of treatment. Larger variations, or differences assessed by examination of large series of patients, may nevertheless give useful information.

Lastly, liver biopsy can provide information as to the presence of complications arising in the course of cirrhosis. Heavy cholestasis, acute portal inflammation, and bile duct proliferation may suggest an element of biliary obstruction, but will usually fail to reveal the site of the obstruction; in a substantial proportion of patients the cirrhosis itself appears to give rise to intrahepatic obstruction. ${ }^{16}$ The chief complication demonstrable by biopsy is primary liver cell carcinoma, and here scintiscanning often enables the biopsy to be taken from a suspect area rather than at random. In the western world the great majority of cases of liver cell carcinoma arises in cirrhotic livers, and the cirrhosis is usually of macronodular or mixed type. ${ }^{17,18}$ Indeed, this fact is one of the justifications for retaining a purely anatomical classification. It is not known whether carcinoma is more likely to arise in macronodular than micronodular cirrhosis because of the passage of time, or because of a greater degree of liver cell hyperplasia in the former, or for some other reason. It is noteworthy in this connexion that in cases of liver cell carcinoma evidence of liver cell hyperplasia in the non-neoplastic tissue is often slight, and in haemochromatosis carcinoma can develop before cirrhosis. ${ }^{19}$

It may be concluded that liver biopsy has a useful part to play in the management of the cirrhotic patient, since it provides information of various kinds, not restricted to the bare diagnosis of the disease. At the same time the problems of sampling error and of present lack of knowledge concerning aetiological factors are such that biopsy should be regarded as part of the 
range of investigations rather than as a final arbiter. It is not uncommon for a liver biopsy to raise a strong suspicion that the patient has cirrhosis, without the diagnosis being fully proven. In such cases a combination of other tests may substantiate or refute the diagnosis, and the role of liver biopsy in cirrhosis is not thereby lessened.

P. J. SCHEUER

\section{References}

'Braunstein, H. (1956). Needle biopsy of the liver in cirrhosis. Arch. Path., 62, 87-95.

'Baggenstoss, A. H. (1966). Morphologic and etiologic diagnoses from hepatic biopsies without clinical data. Medicine (Baltimore), 45, 435-443.

'Wilber, R. D. and Foulk, W. T. (1967). Percutaneous liver biopsy. J. Amer. med. Ass., 202, 53-55.

'Vido, I. and Wildhirt, E. (1969). Korrelation des laparoskopischen und histologischen Befundes bei chronischer Hepatitis und Leberzirrhose. Dtsch. med. Wschr., 94, 1633-1637.

'Rake, M. O., Murray-Lyon, I. M., Ansell, I. D., and Williams, R. (1969). Improved liver-biopsy needle. Lancet, $2,1283$.

'Sherlock, S., Fox, R. A., Scheuer, P. J., and Doniach, D. (1969). Presymptomatic primary biliary cirrhosis. (Abstr.) Gastroenterology, 56, 1222.

'Zeegen, R., Stansfeld, A. G., Dawson, A. M., and Hunt, A. H. (1969). Bleeding oesophageal varices as the presenting feature in primary biliary cirrhosis. Lancet, 2, 9-13.

${ }^{8}$ Rubin, E., Krus, S., and Popper, H. (1962). Pathogenesis of postnecrotic cirrhosis in alcoholics. Arch. Path., 73, 288-299.

'Lee, F. I. (1966). Cirrhosis and hepatoma in alcoholics. Gut, 7, 77-85.

${ }^{10}$ Popper, H., and Schaffner, F. (1968). Hepatic cirrhosis: a problem in communication. Israel J. med. Sci., 4, $1-7$.

"Sherlock, S. (1968). Diseases of the Liver and Biliary System, 4th ed. Blackwell, Oxford.

${ }^{12}$ Scheuer, P. J. (1968). Liver Biopsy Interpretation. Baillière, Tindall and Cassell, London.

${ }^{13}$ Meister, H. P., Szanto, P. B., and Schoolman, H. (1963). Quantitative-morphologic evaluation of postnecrotic cirrhosis. Virchows Arch. path. Anat., 336, 447-464.

1"Popper, H. (1966). What are the major types of hepatic cirrhosis? In Controversy in Internal Medicine, pp. 233243. Edited by F. J. Ingelfinger, A. S. Relman, and M. Finland. Saunders, Philadelphia.

${ }^{18}$ Gall, E. A. (1966). Posthepatitic cirrhosis, fact and fancy. In Controversy in Internal Medicine, pp. 244-256. Edited by F. J. Ingelfinger, A. S. Relman, and M. Finland. Saunders, Philadelphia.

'Datta, D. V., Sherlock, S., and Scheuer, P. J. (1963). Post-necrotic cirrhosis with chronic cholestasis. Gut, 4, 223-230.

${ }^{17}$ MacDonald, R. A. (1956). Cirrhosis and primary carcinoma of the liver. New Engl. J. Med., 255, 1179-1183.

${ }^{18}$ Gall, E. A. (1960). Primary and metastatic carcinoma of the liver. Arch. Path., 70, 226-232.

${ }^{19 B a r r y, ~ M ., ~ S c h e u e r, ~ P . ~ J ., ~ S h e r l o c k, ~ S ., ~ R o s s, ~ C . ~ F ., ~ a n d ~ W i l l i a m s, ~ R . ~(1968) . ~ H e r e d i t a r y ~ s p h e r o c y t o s i s ~ w i t h ~}$ secondary haemochromatosis. Lancet, 2, 481-485. 